\title{
La reforma en Salud Mental en Cataluña: el modelo catalán ${ }^{1}$
}

RESUMEN: Se analiza el estado de la reforma en Cataluña. Se revisa su historia y los diversos recursos existentes. Se extraen conclusiones y se realiza una valoración crítica a partir del documento para la reforma de la Atención Psiquiátrica.

PALABRAS CLAVE: reforma, modelo mixto, cronología histórica, mapa de recursos, análisis comparativo, consecuencias del modelo, valoración.
SUMMARY: We analyse the situation about Catalonien psyquiatric reformation. Revised historie and services now. We have made critic valoration of the document for obteining the following conclusions of psyquiatric atention reform KEYWORDS: Reform, Mixed model, cronologie, services map, comparative analyse, effects of the model, valoration

\section{Prefacio}

Este informe sobre la reforma en salud mental en Cataluna se inscribe en el trayecto que nos propusimos al constituir la asociación autonómica catalana: analizar en la medida que nos fuera posible la red de salud mental en Cataluña, su estado actual, sus líneas de futuro; su finalidad es clara: deseamos contribuir a transformarla.

Advirtamos desde el principio que éste no pretende ser un informe concluso ni mucho menos definitivo sobre un tema tan complejo y vivo, sino una aproximación más o menos acertada sobre el estado actual de la cuestión.

El estado de la reforma en los diferentes territorios del estado español es muy heterogénea y desigual $(1,2,3,4,5)$. Frente a territorios donde las propuestas de la Comisión para la Reforma Psiquiátrica despegaron con gran fuerza -Andalucía $(6,7)$, Asturias (8)-, hasta eliminar los hospitales psiquiátricos en pro de un modelo de atención comunitaria, topamos con otros - Cataluña estaría entre ellos- donde el devenir de la salud mental pareciera no encaminarse en esa precisa dirección.

El modelo mixto de gestión de la sanidad, la persistencia —aunque reordenada y reestructurada - de las antiguas estructuras hospitalarias... han acuñado el concepto de «modelo catalán». Pero este concepto es tan sólo un constructo, pues el modelo catalán tiene una larga trayectoria pasada, un presente complejo que trataremos de analizar y un futuro todavía por venir, aunque parezca ya encaminado.

Rev. Asoc. Esp. Neuropsiq., 2001, vol XXI, n. ${ }^{\circ} 79$, pp. 101-128

${ }^{1}$ Este informe fue presentado en las Jornadas Nacionales de la AEN de Junio de 2001 en Madrid, y ha sido elaborado con la colaboración directa de los siguientes profesionales: Jordi Artigué, Jaume Baró, Regina Bravo, Claudi Camps, Josep Clusa, José García Ibánez, Josep M. ${ }^{a}$ Comelles, Cristina Gispert, Fernando Lana, Isabel Mirones, Angel Pedra, Maite San Emeterio, Jorge Tió, Jorge Tizón y el grupo de trabajo previo (work-shop) que contó con los aportes de numerosos profesionales de la asociación. 
Parece partirse, pues, de una idea previa y prefijada sobre el estado de la reforma en Cataluña. Veremos si es así. Mostraremos lo que sabemos, y no tanto los datos que tenemos sobre los recursos en salud mental catalanes, dificiles de obtener por otro lado, sino el modelo que se dibuja, un mapa representativo de la salud mental en Cataluña. No sólo procuraremos dar cuenta del modelo, sino de lo que nos preocupa más: los efectos que creemos produce.

Intentaremos asimismo matizar y precisar al máximo posible nuestro análisis, pues, aunque el mapa que se dibuja es único, tiene ciertamente posibles lecturas y no todas estarian de acuerdo. Un análisis riguroso, basado en datos y evidencias contrastables, que evite prejuicios y apasionadas opiniones, nos parece el camino más acertado, aun sabiendo que cierta mirada subjetiva se traslucirá.

\section{Cronologia histórica (tabla 1)}

Para empezar a abordar el modelo catalán, iremos primeramente a buscar sus raíces en el tiempo, recordando algunos trazos de su memoria histórica - un recorrido más preciso necesitaría un importante trabajo de indagación-. Como dice Martí Pol, un estimado poeta de nuestra tierra: «Huiría de mí si no pudiera recuperar mi tiempo e interrogarlo... volver al pasado como el que vuelve a la casa donde vivió y, recorriéndola, encuentra todos los indicios de lo que es, y aprende contemplándolos...». Y en esa historia hallaremos algo muy valioso, que quizá hoy no recordamos: Cataluña ha sido una comunidad pionera en el intento de reformar la salud mental.

Estamos en el periodo 1914-1923, y ya la Mancomunidad de las cuatro diputaciones pretende en sus propuestas cierta reforma de la salud mental: mejorar las condiciones de vida de los enfermos, practicar la laborterapia, la hidroterapia o la electroterapia... Poco más adelante, en Barcelona, se constituye la asociación AEN (9), con líneas próximas a la Liga de higiene mental (prevención, tratamiento científico de la enfermedad, etc). Esa traza reformadora la reencontramos en 1931 con la nueva Ley de asistencia psiquiátrica, una avanzada propuesta de sectorización - por comarcas- para crear servicios abiertos en los dispensarios, formar al personal o controlar los internamientos. Aún en 1936, Cataluña lleva a cabo un plan de «Asistencia Psiquiátrica Comarcal» y abre «dispensarios en la comunidad». Es el tiempo de Mira y Lopez, Tosquelles, Vives y Casajuana, y tantos otros. Es un tiempo de esperanza, preludio de una transformación de la salud mental, que se corta abruptamente con la guerra civil.

Deberá transcurrir mucho tiempo, nos encontramos ahora a finales de los años 60, para que nuevamente se palpen vientos de cambio. Por una parte, en 1967, la Diputación se ha propuesto reducir los internamientos sanatoriales y realizar cierta asistencia en la comunidad. Además, están brotando semillas de cambio dentro de los propios muros de los hospitales psiquiátricos de más antigua raigambre: el sanatorio Pere Mata (Tarragona), donde se hacen asambleas de enfermos y se intentan caminos de rehabilitación; el Hospital 
INFORMES

Psiquiátrico de Salt (Gerona), que pretende un proyecto de reforma que resultara finalmente abortado (4); el Instituto Mental de la Santa Cruz y San Pablo (Barcelona) (4) o el Hospital psiquiátrico de San Boi con intentos de transformación desde dentro... son jalones de sobra conocidos por muchos.

En este periodo la Diputación es la encargada de la asistencia psiquiátrica a la patología más grave, y administra los centros de ingreso donde se practica un modelo claramente manicomial (durante mucho tiempo la hospitalización psiquiátrica no se ha contemplado desde Sanidad, sino como beneficencia desde la Diputación). Coexiste con la saturada asistencia que se realiza en las plazas de neuropsiquiatría de la seguridad social para los casos no tan severos, en las que se practica un modelo psiquiátrico con tiempos de atención muy restringidos y respuestas básicamente farmacológicas.

Y prosiguen los intentos, nuevos y no conseguidos intentos desde la administración; así, el Plan de Asistencia psiquiátrica de 1975 de inspiración comunitaria que se basa en la psiquiatría de sector francés... Se intentarán cambiar algunas formas, reordenar las estructuras existentes, sectorizar los recursos, pero el fondo del anterior modelo de atención persistirá incólume, mostrando así la dificultad de modificarlo.

Es entonces, estamos a mediados de la década de los 70-80, que en Cataluña se impulsan esforzados proyectos reformistas desde diversos ámbitos. Así, muchos profesionales que disentían del modelo que se practicaba abren los primeros Centros de Higiene Mental, lugares pioneros de la reforma en salud mental. Son centros de carácter comunitario, preventivo, que otorgan gran peso a lo psicoterapéutico y que intentan realizar su labor en la propia comunidad (contactan con las asociaciones de vecinos, con las escuelas, con los médicos de primaria, cobran precios populares, se nutren del psicoanálisis y la antipsiquiatría...). Con esta misma intención, también algunos Hospitales psiquiátricos -Reus, Gerona - abren Dispensarios Comunitarios en diferentes comarcas con una idea de sectorización; son incipientes equipos formados por psiquiatra y asistente social. Igualmente, algunos profesionales comprometidos procuran reformar desde dentro los propios servicios de neuropsiquiatría.

A inicios de los 80 estos núcleos de cambio confluyen para dar lugar a los primeros Centros de Atención Primaria èn salud mental (CAPS), que nacen con la idea de ser el eslabón primario de atención para la salud mental.

La propia Diputación promueve esta corriente de cambio y procura sentar las bases de un modelo reformista. En este sentido, realiza unas Primeras jornadas sobre la ordenación de la asistencia psiquiátrica en Cataluña en Noviembre del año 1985 (10), en las que una gran cantidad de profesionales de diferentes disciplinas relacionadas con la salud mental exponen sus ideas y propuestas. Por este tiempo ya funcionan algunos centros de día. También han surgido los primeros hospitales de día (Torre de la Creu, CPB) y comunidades terapéuticas que beben en las fuentes de la psiquiatría comunitaria. 
En 1981 se asumen las transferencias de la Seguridad Social y se crea el Mapa sanitario $^{2}$. También se crea un plan de reordenación hospitalaria. Paulatinamente, especialmente a partir de 1985, se van abriendo nuevos centros: centros de atención infanto-juvenil, centros de atención para drogodependencias..

Por este tiempo los hospitales psiquiátricos han ido modificando algunas instalaciones y han diferenciado sus unidades en agudos, subagudos y media y larga estancia (separando los pacientes psiquiátricos menores y mayores de 65 años y los diagnosticados de retraso mental).

Así pues, el llamado modelo catalán ancla sus raíces en diversos intentos reformistas, intentos que desde 1985 , como en el resto del estado, quedan claramente influidos por el documento para la Reforma de la Asistencia Psiquiátrica de 1985 (11) y la Ley General de Sanidad de 1986. Este documento es la base de partida clave en la evolución de la salud mental en el territorio espafiol. Sus puntos de referencia son claros y precisos, y nos servirán para sostener nuestro análisis:

Procurar que la atención en salud mental sea considerada en el mismo rango que otras especialidades, incluyéndola en la asistencịa sanitaria general; basar la atención en salud mental en la atención primaria de salud como primer eslabón básico, con la atención especializada en segundo término asesorando al equipo básico, realizando seguimientos de pacientes y formando profesionales; procurar la recuperación y reinserción en la medida de lo posible de los enfermos; garantizar sus derechos civiles; desarrollar programas de rehabilitación activos; reducir la hospitalización psiquiátrica a lo imprescindible y hacerlo en unidades de agudos de hospitales generales; reconvertir y superar el hospital psiquiátrico (reducir camas, externar pacientes con programas de rehabilitación y reinserción social, reciclar sus profesionales...); desarrollar programas de prevención; poner especial peso en la atención a la infancia, geriatría, drogodependencias y psiquiatria penal; procurar el seguimiento de los enfermos de cierta cronicidad en su entorno socio-familiar con residencias apropiadas y programas rehabilitadores...

En suma, se trata de situar la atención en salud mental en una forma de atención moderna que otros países ya desarrollan, una atención basada en redes de recursos sanitarios y comunitarios que se aleja clara y decididamente de los modelos que confinaron a los enfermos en lugares de exclusión y cuyas formas de atención giraban alrededor del hospital psiquiátrico, una «institución total» que con su función residencial y custodial sostenía y ordenaba entre sus muros toda la vida de los internados.

El antiguo modelo atendía a los pacientes más graves en el recinto hospitalario sin apenas posibilidades de rehabilitación activa ni de reinserción en la comunidad o bien mediante recursos de atención sanitaria que utilizaban un modelo psiquiátrico tradicional destinado a la resolución de los síntomas (así, las consultas neuropsiquiátricas). Mientras, el nuevo paradigma de atención pretende una atención en salud mental inserta en la 
INFORMES

comunidad que procura sostener y tratar al enfermo, incluso el más grave, en redes de recursos sanitarios y socio-comunitarios interconectados (gráfico 1). El modelo de la reforma sostiene así que la enfermedad mental puede ser tratada y rehabilitada como cualquier otra enfermedad, sin necesidad de mantener y tratar a las personas afectas en lugares de exclusión.

Tras ese largo periodo llegamos a un momento decisivo, crucial a nuestro entender: en 1990 se inicia el traspaso de competencias de las diputaciones al gobierno catalán, a la Generalitat. Punto clave, que va a marcar la siguiente etapa, con la que llegaremos a nuestros días.

La Generalitat, gobernada por Convergencia i Unió, asume en 1991 las competencias de Tarragona, Lérida y Gerona (de Barcelona asume la gestión, sin que los traspasos hayan quedado aún totalmente realizados) y crea dos elementos básicos: el Servei Català de Salut y el Pla de Salut mental. Se inspira en la LLOSC, la Ley General de Ordenación Sanitaria que ha aprobado el Parlament Català y cuyo plan es integrar los recursos de salud mental en la asistencia general (12).

Pero ese Pla de salud mental rápidamente muestra un carácter particular que repite el modelo aplicado a la Sanidad general (a la red de hospitales generales, la XHUP). Defiende lo que se ha venido en llamar modelo mixto ${ }^{3}$ : el programa de salud mental ya no provee los recursos como acostumbra el sistema público de salud, sino que tiene como finalidad la planificación, financiación, contratación y valoración de los recursos de salud mental, recursos que pueden provienir de lo público (Institut Català de Salud (ICS), Diputaciones, Ayuntamientos) o de lo privado (13).

Este punto es decisivo: se introduce en este momento un modelo de gestión empresarial privatizador para los recursos de la sanidad pública $(14,15)$. Decisivo no sólo por los modelos organizativos que genera y por el espíritu que introduce en el ámbito clínico, tan delicado, sino por los efectos que esto va a desencadenar. A partir de este momento muchos recursos de la salud mental se impregnarán de este modelo gestor: las grandes empresas del sector (en su mayoría las que gestionan los hospitales psiquiátricos) adquieren mayor peso, las pequefias empresas proveedoras se unen para formar organismos de gestión de superior alcance y los profesionales que han llevado el peso del proyecto reformista deben dedicar su atención a la gestión de recursos.

Las directrices de este plan son claras: eficacia y eficiencia empresarial, ahorro económico, objetividad, fomentar la sana competencia, introducir criterios de validación... Al no estar el Servei Català de Salud implicado en la gestión de un servicio, sino que desde fuera valida sus resultados, la propuesta del «modelo mixto» parece posible. Pero en la práctica las cosas no son así: existe una gran heterogeneidad de recursos, ya que los proveedores son muy variados; se siguen generando grandes gastos, pues es muy difícil

${ }^{3}$ El desarrollo de este modelo de gestión de la sanidad no se puede atribuir sólo a un determinado gobierno, pues es el resultado de complejos entramados político-administrativos de larga historia. 
INFORMES

contener y frenar el ingente gasto hospitalario y farmacológico sin una decidida voluntad más allá de criterios economicistas ...

Poco a poco, al introducir criterios economicistas y mercantilistas en el sector sanitario, la atención pública se va transformando: los centros de atención se convierten en «empresas proveedoras», los recursos de atención en «cartera de servicios» y los pacientes o usuarios a menudo en «clientes». La lógica empresarial empieza a impregnar muchos eslabones de la salud mental, marcando y acortando la duración de la atención (las altas hospitalarias, los periodos en los recursos intermedios; las visitas en los Centros de salud mental), incrementando la burocracia y los items cuantificadores, creando el temor a posibles competencias entre los proveedores, etc. Además, el programa de salud Mental trata de obtener los mayores rendimientos y el máximo incremento de los recursos sin aumentar demasiado la inversión.

A pesar de este modelo mixto de gestión economicista, los traspasos van a tener efectos beneficiosos en el sector: se abren más centros de salud mental de adultos y de infanto-juvenil (CSMAs y CSMIJs) para asistir a toda la población, se prosigue con mayor empeño la reconversión de las antiguas plazas de neuropsiquiatría en atención primaria que irán siendo absorbidas por los centros de salud mental, se inicia la reforma de la atención primaria para ampliar el tiempo de atención y su calidad, se amplían el número de centros de día (que en 1991 se constituye como red) y el de hospitales de día, se crea el trabajo en circuito para integrar los diferentes niveles asistenciales de primaria, secundaria y terciaria... A la vez, y respondiendo en muchas ocasiones a lo que produce más alarma social, aparecen Unidades especializadas: Unidades para Trastornos alimentarios, Unidades de Sueño, Programas para Ludopatías, para TOC (Trastorno obsesivo-compulsivo), para Esquizofrenias refractarias...

Con mucha mayor lentitud, se amplían los recursos destinados al Trastorno Mental Grave o Severo, en muchos casos por presiones de ciertos colectivos; la Federación de Asociaciones de familiares de enfermos mentales agrupa más de 3.000 familias y tiene un fuerte poder mediático. Se crean entonces los Programas de Atención al Trastorno Mental Severo (16), en el cual cada paciente dispondrá de un profesional de referencia, un Proyecto terapéutico individualizado (el PTI) y un Programa de Rehabilitación Individualizado de Rehabilitación y Reinserción (PIRR). Todo ello incluye seguimiento de pacientes, información a las familias, atención domiciliaria, mejora de la atención en crisis, importancia de la rehabilitación y reinserción, coordinación con el circuito... Este programa se irá instalando paulatinamente en los diversos centros de Atención. Se intentan también líneas de reconversión de camas hospitalarias, solicitando a los Hospitales psiquiátricos la diferenciación de sus pacientes en unidades especificas (MILLE o larga estancia, agudos, subagudos...) o temáticas (disminuidos, psicogeriatría...). Eso permite que ciertos recursos, así los destinados a esos dos últimos colectivos, dependan de otros presupuestos: Disminuidos de Bienestar Social y Psicogeriatría de un programa de más alcance que Sanidad destina a los de mayor edad, el programa «Vida als anys». 
Hoy existen diversos proyectos sobre los servicios de psiquiatría y salud mental que señalan caminos futuros para la salud mental en Cataluña. Uno de los más señalados es el de reordenación y reconversión de los hospitales psiquiátricos monográficos, los hospitales más antiguos (17). Con este fin, el Programa de Salud Mental ha llegado a un acuerdo en el año 1999 con varias entidades proveedoras para que inviertan en los cinco próximos años 7.600 millones, que el Programa se compromete a devolver con intereses en un plazo de 20 años. Este proyecto consiste en la creación de 5 nuevas Unidades de Agudos (que deberán estar fuera del hospital psiquiátrico), 7 unidades de Alta Dependencia Psiquiátrica, 2 de Patología Dual, 1 de Patología Dual con Alcoholismo, 1 de Psiquiatría Penitenciaria, 1 de Alta Seguridad, 1 de Subagudos para Adolescentes y 2 Unidades Polivalentes en la comunidad. Estas Unidades de nueva creación se construirán tanto en el interior de los hospitales (alguna ya ha empezado a funcionar), como en la comunidad (así, las Unidades Polivalentes, que incluyen centro de día, hospital de día y recurso residencial). Otros proyectos, a corto o largo plazo, están en la línea de establecer todas las unidades de agudos en hospitales generales (18), desarrollar programas específicos de psicoterapia, desarrollar nuevos programas para el Trastorno Mental Severo (programa $\mathrm{PSI}^{4}$ ), mejorar los recursos rehabilitadores, etc.

También en el momento presente, y desde Bienestar Social en este caso (19), se están intentando desarrollar desde hace muy poco, año 2000, recursos comunitarios: pisos protegidos, residencias comunitarias, plazas de trabajo protegido, clubs socio-terapéuticos... Destaquemos ya este punto: la escasez de posibilidades de reinserción en la comunidad es una de las mayores debilidades del modelo y uno de los que más efectos produce, pues incide en todos los niveles, tal como veremos más adelante.

Igualmente, cabe destacar las muchas formas de intervención que se realizan en numerosos ámbitos; así, a nivel infantil la coordinación con justicia juvenil, con los equipos de asesoramiento de infancia y adolescencia (EAIA) de la Dirección General de Atención al Menor (DGAM), con el programa CTIC (de coordinación de recursos para la infancia), con los equipos de asesoramiento psicopedagógico (EAP) o con la red SAP de centros de atención precoz... y, a nivel de adultos, con prisiones, con la red UFIS para demencia y psicogeriatría, con la red de CAS, así como la interconsulta con Servicios Sociales de atención primaria, etc.

La culminación de ese largo proceso ha sido el decreto-ley que define la Red de salud mental de utilización pública en julio de 1999 (20). Su finalidad es integrar, en una red única, todos los servicios que realizan la atención en salud mental; aunque ese decreto ya advierte que será de implantación progresiva, dada su complejidad. Su inspiración, la letra de la ley, reza así: conseguir un modelo de atención interdisciplinar e intersectorial (con la participación de recursos sociales, educativos); garantizar la continuidad asistencial; procurar la atención y reinserción en la comunidad; dedicarse en especial a la atención infanto-juvenil y a los trastornos mentales graves y severos; atender las necesidades de las familias; conseguir criterios de calidad.

\footnotetext{
${ }^{4}$ El programa PSI, Plan de Servicios Individualizado, es un programa piloto basado en el modelo de gestión de casos con un coordinador de proyecto individual (CPI) que atiende a cada enfermo, trabajando en su entorno y coordinando todos los recursos en juego.
} 
El decreto, que no propone programas ni dotaciones mínimas para los recursos de salud mental, se compromete a prestar los siguientes servicios: atención especializada (red de CSMAs y CSMIJs), servicios de hospitalización de agudos (independientemente, se afirma, de su ubicación física), servicios de subagudos, servicios de internamiento en la comunidad (así, las comunidades terapéuticas), de atención específica (las de atención a anorexias, ludopatías, TOC, etc.), de hospitalización parcial (los hospitales de día), de rehabilitación comunitaria (los centros de día), unidades polivalentes (que incluyen varios servicios a la vez).

El decreto señala también que todos estos servicios deberán, además de sus funciones específicas, promocionar la salud, educar a la población, realizar medicina preventiva, desarrollar investigación clínica y epidemiológica e impartir docencia.

Llegamos aquí al punto que tratamos de analizar: cuál es el estado de la reforma en Cataluña?, ¿existe una auténtica red de salud mental (pues no basta con decretarla para que aparezca)?, ¿qué modelo de atención se configura?, ¿cómo está dotado?, ¿cuáles sus fortalezas y debilidades?, ¿hacia dónde se dirige?.

Para perfilar con la mayor claridad estos interrogantes, analizaremos uno por uno, desglosándolos, los diferentes niveles de atención, advirtiendo antes de una dificultad añadida para este análisis: los datos han sido conseguidos desde múltiples ámbitos, con algunas dificultades algunos, aunque conviene puntualizar que los datos sobre recursos han sido suficientemente contrastados. Otra dificultad es la calificación de los recursos; así, recursos muy diversos, algunos bastante limitados, pueden tener la misma denominación.

Análisis de recursos (ver mapa de recursos de tabla 2)

Para evaluar algunos de estos recursos utilizaremos las estimaciones propuestas por diversos comités de expertos, en especial las del Cuaderno técnico de AEN denominado «Hacia una atención comunitaria de calidad», que basa sus estimaciones en diferentes programas de Salud Mental nacionales e internacionales (21).

Recursos sanitarios y comunitarios (en relación con el Departamento de Sanidad) (17) Centros de salud mental $(22,23,24)$. Actualmente hay una red de 66 CSMA y 33 CSMIJ, pronto serán 67 y 34 respectivamente, que cubre zonas entre 10-20.000 habitantes hasta $250.000 \mathrm{~h}$ o más; el promedio es de 100-150.000 habitantes. Sus módulos de atención ${ }^{5}$ son de $1-2 \mathrm{~h} / 1000$ habitantes en CSMIJ y 2-3 h/1000 habitantes en CSMA ${ }^{6}$.

En todo caso, pese a que esa red se ha ampliado a todo el territorio catalán, el incremento de la demanda, cifrado entre 1'5 hasta 2-3 veces (pues depende de zonas) la de años pasados, ha desbordado con creces las posibilidades de atención que antes eran posibles. En parte, se ha debido a la absorción de los pacientes de los cupos de neuropsiquiatría; por

${ }^{3}$ El modulo de atención es el número de horas totales a la semana que destina un centro de salud mental para un determinado número de habitantes (por ejemplo, un módulo de $3^{\prime} 3 \mathrm{~h} / 1000$ hab. correspondería a 330 horas de atención para 100.000 habitantes repartidas entre varios profesionales).

${ }^{6} \mathrm{La}$ ratio de calidad es de 3-4 horas por 1000 habitantes como mínimo $(21,23)$. 
INFORMES

otro, al trabajo con la atención primaria y la sensibilización de los médicos de cabecera. Pero ese creciente incremento no se ha acompañado de los correspondientes aumentos del personal (inferiores en general al $70 \%$ : entre 10-65\%).

Abordajes psicoterapéuticos: hay tan sólo dos unidades específicas para todo el territorio que realizan tratamientos psicoterápicos, psicoterapias breves de orientación psicoanálitica a tiempo limitado. Se trata de un unidad para adultos (PTA) y otra para infanto-juvenil (PTI) situadas en Barcelona ciudad, cuyos pacientes son derivados en un $75 \%$ por los Centros de salud mental. Un estudio realizado por estas unidades confirma la mejoría clínica de un elevado tanto por ciento de los pacientes tratados y la importante disminución o retirada de los tratamientos farmacológicos que estos tomaban.

Más allá de esta posibilidad, estos abordajes dependen del voluntarismo de los profesionales de atención especializada. Cabe añadir la persistencia de algún antiguo centro de higiene mental no reconvertido que sigue atendiendo con técnicas psicoterapéuticas y a precios módicos con la ayuda de algún Ayuntamiento (Cornellá, Badalona).

Programa TMS (16): éste es un programa con el que se ha dotado a los centros de salud mental (7.900.000 pts) para la atención integral al paciente severo y que muy pronto se extenderá a todos los centros de salud mental. Su ambición es tratar integralmente a este tipo de pacientes, con mucho mayor apoyo de enfermería y de trabajo social, y el Programa de Tratamiento Individualizado (PTI) antedicho. Su dotación, sin embargo, no alcanza a cubrir a todos los pacientes de estas características ni con la calidad requerida. A veces sólo permite seguimientos farmacológicos y controles de algunos factores de autonomía personal de los enfermos, ciertí intervención educativa de las familias, visitas domiciliarias muy delimitadas y poco más. Su cumplimiento es muy variable, ya que depende de los criterios de cada centro.

Las consecuencias de tamaño incremento de la demanda de los CSMA y CSMIJ han sido la disminución del tiempo de atención por visita, el aumento del tiempo de espera para primeras y segundas visitas, el consiguiente aumento de los tratamientos farmacológicos (se han incrementado los antidepresivos en un $40 \%$ y las benzodiacepinas en un 10 $\%$ por lo menos) y la drástica reducción de abordajes más psicoterapéuticos antes posibles.

Red de recursos para toxicomanias (25): está constituida por 54 centros de atención (CAS), por 10 Unidades de Desintoxicación hospitalarias y dispone de 11 comunidades terapéuticas (la mayoría privadas financiadas). Funciona actualmente separada de la Red de salud Mental y tiene presupuesto y programa propio. Realiza diversos programas de atención y de tipo preventivo. Cabe destacar de esta red la imbrincación de recursos provenientes de parroquias (en sus inicios), ONGs, comunidades religiosas y otros. La alarma social que estos enfermos generan ha movilizado muchos recursos, que más recientemente han dado lugar a la creación de programas para Patología Dual y Unidades especializadas de reciente puesta en marcha. 
Red de centros de día. Hay actualmente 42 centros de día para adultos y 7 centros de día para infantil. En total 49 centros de día en toda Cataluña (pronto serán 52). Desarrollan programas específicos según lugares y posibilidades (programa funcional de actividades, de atención a familias, de inserción comunitaria, de soporte a pisos asistidos, de inserción laboral o de externación de pacientes de larga estancia). Su dotación es muy variable, con profesionales de muchos tipos (algunos monitores tiene únicamente categoría de auxiliares de clínica) y acostumbran a estar infradotados de personal (alguno sólo tiene dos o tres profesionales). Los Centros de Día infantiles están en general ubicados en antiguas escuelas especiales. Su distribución geográfica es muy irregular. Hay actualmente 1409 plazas, o sea, unas 23 plazas para cada 100.000 habitantes ${ }^{7}$. Para paliar los déficits, muchos de esos centros tiene diversas modalidades de vinculación (algunos amplían número de pacientes atendidos a costa de disminuir sus posibilidades rehabilitadoras).

Esos centros se insertan en redes comunitarias más amplias y procuran realizar técnicas de rehabilitación activa. Sin embargo, la infradotación de estos recursos, así como la escasez de recursos comunitarios de inserción (laborales, de vivienda, sociorecreativos, etc.), ha traído como consecuencia la posibilidad que muchos de los centros de día no puedan tener más función que la de «guardadores» de pacientes, en general de pacientes cronificados y con pocas posibilidades de rehabilitación activa (un grupo de trabajo sobre Rehabilitación del Servei Català de Salut (25) ha calculado que tan sólo llega a un 5-10 $\%)$. Es significativo que se siga llamando «centro de día» lo que en otras comunidades recibe el nombre de Unidad de rehabilitación.

Hospitales de dia. Existen actualmente 8 hospitales de día para adultos (7 en Barcelona provincia, de ellos 3 Barcelona capital, uno en Lérida en el hospital general y ninguno en Tarragona y Gerona), 6 de infanto-juvenil y 2 de adolescentes. Señalar que algunos de ellos consisten en un número de camas de un hospital general. Nuevamente cabe resaltar que la infradotación de recursos ha producido, por un lado, el acortamiento de las estancias (hace pocos años tenían un promedio de 8-12 meses, hoy es de 3-6 meses como máximo) y, por otro, la creciente dificultad para su labor rehabilitadora. Hay actualmente 276 plazas de hospital de día de adultos y 124 de infanto-juvenil, es decir, unas 4'6 plazas de adultos para 100.000 habitantes y 2 de infanto-juvenil ${ }^{8}$.

Comunidades terapéuticas. Sólo hay tres en la red catalana (también todas en Barcelona).

Cabe resaltar de todas estas redes de recursos antedichos que pocas pueden tener un funcionamiento propiamente comunitario, aquel donde los recursos sanitarios (CSM, centro de día, hospital de día, comunidad terapéutica) se insertan en la comunidad con recursos de inserción (taller protegido, pisos asistidos).

${ }^{7} \mathrm{La}$ ratio de calidad para centros de día es mínimo 20 y óptimo 50 plazas para 100.000 habitantes (21).

${ }^{8}$ La ratio de calidad para hospitales de día es de 10-19 plazas de adultos y 3-5 de infanto-juvenil para cada 100.000 habitantes (21) 
INFORMES

\section{Recursos hospitalarios}

Hospitales psiquiátricos: un estudio del año 1975 realizado en todo el territorio español (4) hablaba de 17 hospitales psiquiátricos en el territorio catalán; algún otro lo elevaba a 21 (13 Barcelona, 2 Gerona, 2 Lérida y 4 Tarragona) con una población de unos 11.000 pacientes. El Programa de salud Mental (17) menciona que actualmente (año 2000) existen 19 centros psiquiátricos, de ellos 7 hospitales monográficos. Y diferencia la atención entre pacientes agudos, subagudos y de larga estancia (MILLE). Como centro psiquiátrico entiende todo centro de carácter hospitalario que esté autorizado a realizar internamientos psiquiátricos, siendo los monográficos los hospitales destinados en exclusiva a este tipo de atención. Los hospitales monográficos son los hospitales psiquiátricos más antiguos y pertenecen a algunos de los principales proveedores del Programa de Salud Mental: 1 en Gerona (Parc Martí Julià, antiguo psiquiátrico de Salt), 2 en Tarragona (Instituto Pere Mata y Sanatorio Villablanca), y 4 en Barcelona provincia (San Juan de Dios y Benito Menni de San Boi, Sagrado Corazón de Martorell y Recinto Torribera de Santa Coloma). Señalemos que muchos de los servicios del territorio (centros de día, CSMAs y CSMIJs), sobre todo en Tarragona, Lérida y Gerona, son de la empresa que gestiona el hospital psiquiátrico monográfico de la provincia.

Hoy hay 3325 camas para crónicos (MILLE) con un $25 \%$ de alta dependencia, 394 para subagudos (entre monográficos, unidades polivalentes y hospitales de día) y 788 para agudos (entre hospitales monográficos y hospitales generales). No se incluyen psicogeriatría ni disminuidos.

En los últimos tiempos se ha producido una importante disminución de las camas en los hospitales psiquiátricos: de casi 5.000 en 1992 se ha pasado a las 3.800 actuales. Esta disminución ha sido posible en gran manera merced a la reconversión de recursos, pues muchos de esos pacientes han pasado a depender de otros programas (Psicogeriatría de recursos socio-sanitarios y Disminuidos de Bienestar Social), aunque en ocasiones dichos pacientes hayan seguido permaneciendo en el mismo Hospital Psiquiátrico. Un ejemplo: en el Parc Hospitalari Martí i Julià, del total de 430 camas, 200 son de la Unidad de Psicogeriatría y 60 de la Unidad de Retraso mental, es decir, la mitad aproximadamente.

Destaquemos asimismo que, debido a la falta de recursos que también afecta a esas unidades hospitalarias y a la presión de la administración, los ingresos psiquiátricos se han ido reduciendo en duración, con el consiguiente efecto de «puerta giratoria», donde demasiadas veces los pacientes salen casi igual a como entraron.

Una gran mejora, pese a las carencias aún existentes, ha sido el incremento en más del doble de las plazas de hospitalización infantil: 48 camas entre URPIs (Unidad de referencia psiquiátrica infantil) y UCAs (Unidad de contención de adolescentes), unas

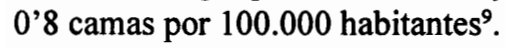

\footnotetext{
${ }^{9}$ La ratio de calidad para Unidades de Hospitalización infantil y de adolescentes es de 1-2 plazas y 2-3 plazas respectivamente por cada 100.000 habitantes (21).
} 
INFORMES

Los hospitales monográficos han seguido dos caminos: mientras unos se han intentado reformar abriéndose al exterior y creando recursos de tipo rehabilitador (han reducido camas y creado unidades polivalentes en la comunidad, por ejemplo), otros han mantenido un modelo tradicional hospitalario de tipo más cerrado.

De todos modos, el Programa de Salud Mental ha puesto en marcha el proyecto anteriormente citado de reconversión y reordenación de los Hospitales Psiquiátricos Monográficos. Así pues, dichos Hospitales persistirán, pero deberán diferenciar más la población que atienden, establecer programas específicos para determinadas patologías y procurar que la atención que realizan para los pacientes agudos se realice en dispositivos cercanos a la comunidad.

Atención de agudos en hospitales generales.

Hay varios hospitales generales que tienen servicios de urgencias y camas de media estancia, sobre todo en Barcelona provincia (Clínico, San Pablo, Bellvitge, Hospital del Mar; Valle Hebrón, S. Gervasio). Atienden la población que acude espontáneamente por situaciones de urgencia y algunos todavía mantienen consultas externas.

Muchos de esos servicios no pertenecían propiamente a la red asistencial, y funcionaban autónomamente respecto al entramado de centros de salud mental, por lo que hoy se está intentando integrarlos a la red de salud mental de una forma más clara. El problema más preocupante es que muchas veces la atención a los pacientes sigue las líneas de investigación del momento (tienen Programas específicos de Patología Dual, Esquizofrenia Refractaria, TOC, etc.), muchas destinadas a ensayos de tipo farmacológico, que dejan a los pacientes temporalmente fuera del circuito asistencial.

Hoy está en proyecto (18) depositar la mayor parte de la atención para pacientes agudos en hospitales generales (en Barcelona parece ha sido por presiones del Ayuntamiento). Muchas de las nuevas unidades de agudos seguirán perteneciendo a los Hospitales Monográficos .

Atención urgente: No hay actualmente en el territorio catalán un dispositivo de atención domiciliaria en crisis, salvo el de los Programas de Trastorno Mental Severo. Conviene destacar en Barcelona ciudad y cercanías la Unidad de crisis del Instituto Psiquiátrico Municipal; y también el servicio de urgencias del 061, que atiende a domicilio muchas urgencias psiquiátricas y que posee desde hace un tiempo la potestad de realizar traslados involuntarios para que el Hospital de referencia valore la posibilidad de un ingreso involuntario, un tipo de ingreso que se ha incrementado en los últimos años. Actualmente se están probando dispositivos de atención telefónica urgente para que consulten los profesionales de primaria y los hospitales sin servicio de psiquiatría. En los demás territorios la atención urgente corre a cargo del médico de cabecera. 
INFORMES

Recursos comunitarios (en relación con el Departamento de Bienestar Social y el de Trabajo)

Pisos asistidos y residencias. Es destacable en la salud mental de Cataluña el escaso desarrollo de este tipo de recursos. Hasta el año 2000 no se había puesto en marcha ningún plan específico. Hoy hay un plan desde Bienestar social (19) para empezar a desarrollarlos en el que reconoce existen aproximadamente unas 10.000 personas con disminución debida a enfermedad mental (de ellas un $85 \%$ con diagnóstico de esquizofrenia) y que un $40 \%$ de los «sin techo» padecen enfermedad mental.

Se sabe que existen 60-70 plazas de pisos asistidos (a los que se podrían sumar otras 100 de pisos asistidos aún no concertados) y 100-130 plazas de residencia pertenecientes a Bienestar Social en toda Cataluña; estos recursos están, hoy por hoy, sobresaturados por la importante demanda y necesidad existentes. Se estima que en 1-2 años pudieran abrirse 200 más en diferentes zonas del territorio (Sabadell, Tarrasa, Dreta Eixample, Tarragona, Igualada, Manresa, Vilanova i la Geltru). Hay pues 2'6 plazas de pisos para cada 100.000 habitantes y 2 ' 1 de residencias ${ }^{10}$.

Ese mismo estudio de Bienestar Social realiza una estimación de necesidades de 930 plazas de hogar-residencia y 1044 de pisos protegidos. Estamos, pues, entre un 10 a $15 \%$ de las necesidades. La gran mayoría de enfermos debe vivir con sus familias o en residencias para $3^{a}$ edad o formar parte de los «sin techo». Señalemos que Bienestar Social gestiona de forma centralizada estos recursos y que los enfermos con menor grado de disminución y que disponen de pensiones deben aportar parte de éstas para disponer de una plaza.

Trabajo protegido. Se calcula que en toda Cataluña hay como máximo unas 150 plazas. La mayoría de ellas están destinadas a enfermos mentales bien conservados y con buen nivel de autonomía. Algunas pertenecen a las Asociaciones de familiares o a los escasos recursos comunitarios que existen ( $\mathrm{Pi}$ i Molist, Joia, Tres Turons). Hay pues 2'5 plazas de trabajo tutelado para cada 100.000 habitantes ${ }^{11}$. Así, una gran parte de esta población de enfermos queda fuera de dicha posibilidad.

Clubs socio-terapéuticos. Apenas existen, aunque Bienestar Social subvenciona 3 como experiencia piloto y proyecta abrir más. Los otros que existen pertenecen a las Asociaciones de familiares o forman parte de alguno de los recursos intermedios.

Redes de voluntariado. No se sabe que existan como tales, por lo menos especialmente dedicadas a este tipo de enfermos, salvo las de algún hospital psiquiátrico (San Juan de Dios de San Boi) o de las Asociaciones de familiares.

10 La ratio de calidad tanto para pisos asistidos como para residencias es de 20 plazas para cada 100.000 habitantes como mínimo y 50 como óptimo (21).

${ }^{11}$ La ratio de calidad es de 15 plazas de trabajo protegido para 100.000 habitantes como mínimo y 30 como óptimo (21). 


\section{Nivel de los profesionales (23)}

En la red se ha incrementado notablemente la presencia de psiquiatras (en un 30-50\%), mientras los psicólogos han aumentado poco (1-10\%) y los trabajadores sociales apenas, cuando no han disminuido, especialmente a nivel hospitalario. El aumento de la enfermería en S. M (50-100 \%) ha sido gracias a que antes casi no existía. En los equipos multidisciplinares, si tenemos en cuenta las proporcionalidad aconsejada por comites de expertos para cada tipo de profesional, los psiquiatras realizan ahora más horas de atención en relación con los profesionales de otras titulaciones (en CSMA las horas de psiquiatra es de un $41 \%$ frente a un $20 \%$ de psicólogo, $11 \%$ de enfermería y $12 \%$ de trabajo social; y en CSMIJ es de $24 \%, 48 \%, 3 \%$ y $8 \%$ respectivamente para Pt, Ps, Enf y Ts) ${ }^{12}$.

No existe una reglamentación homogénea laboral en el sector, pues el Programa de Salud Mental lo deja al albur de los centros proveedores. Esto implica que los sueldos de los profesionales tengan grandes variaciones de unos lugares a otros (puede ser incluso el doble). Los profesionales de salud mental no tienen incentivaciones profesionales ni complementos de exclusividad (predomina el pluriempleo, sobretodo el trabajo en consulta privada), recibiendo en general sueldos más bajos que en el resto de España. Tampoco hay una reglamentación laboral respecto de los coordinadores de los distintos servicios, siendo mayoría de psiquiatras en los CSMA y de psicólogos en los CSMIJ y centros de día.

\section{Presupuesto de salud mental (gráfico 2)}

El análisis del presupuesto en Salud Mental nos ayuda también a dibujar el modelo de atención.

El presupuesto en Salud Mental del año 2000 tiene varias fuentes: del Programa de Salud Mental (18.300 millones de Sanidad, con la previsión de llegar casi a 20.000 en el próximo), de la Psiquiatría de los Hospitales Generales (4.000 millones de Sanidad), de Psicogeriatría (4.400 millones que dependen de Programas Sociosanitarios), de la Red de Drogodependencias (cuyo presupuesto de 2.700 millones proviene de Sanidad y una parte del Plan Nacional de Drogas) y del presupuesto que Bienestar Social destina a la Red de Atención al Retraso Mental (10.500 millones) o a recursos comunitarios (no precisada).

La Salud Mental constituye el 4'07\% del presupuesto total de Sanidad (738.000 millones), si sumamos las partidas más claramente destinadas a este apartado (Programa de Salud Mental, Psiquiatría de Hospitales generales, Psicogeriatría y Drogodepencias).

A su vez, el Programa de Salud Mental reparte su presupuesto entre los diferentes recursos existentes, con proporciones muy variables, sobresaliendo el $35 \%$ que destina a Larga Estancia frente al $26 \%$ para centros de salud mental, $7 \%$ para centros de día y $6 \%$ 
INFORMES

para hospitales de día, los recursos comunitarios con más posibilidades de rehabilitación y reinserción. También destaca el presupuesto total que destina a recursos hospitalarios (un 55-60\% cómo mínimo, si contamos el $35 \%$ para MILLE, el $8 \%$ para subagudos y el $13 \%$ para agudos).

\section{Análisis valorativo}

¿Cuál es, pues, el estado de la reforma en salud mental en Cataluña? Estimamos que se ha avanzado mucho en los últimos 25 años en la atención en salud mental como hemos podido comprobar y que se han efectuado numerosas reformas, pues ciertamente los pacientes son atendidos con mayor cuidado, hay muchos más recursos terapéuticos y programas de calidad, destacando en especial el desarrollo de la atención infanto-juvenil y de adultos, la integración de la salud mental en la atención sanitaria general, los programas para asesorar a la atención primaria, la reforma (aún no terminada) de los ambulatorios de atención primaria, la creación de plazas de recursos intermedios, los programas TMS, la disminución de camas psiquiátricas reconvertidas en otras (como psicogeriatría) o en recursos rehabilitadores (así, unidades polivalentes en la comunidad), la propuesta de que los ingresos se realicen en unidades de agudos fuera del hospital psiquiátrico, etc.

Sin embargo, a nuestro entender, no se ha puesto suficiente acento en las líneas más fuertes que propugnaba la comisión para la reforma (11), las que supondrían una auténtica transformación del antiguo modelo. Así encontramos (gráfico 3):

- Persiste en buena medida la discriminación de la atención a la salud mental en el Sistema General de Salud (el presupuesto destinado a Salud Mental es aún muy bajo).

- La atención en salud mental sigue consumiendo gran cantidad de recursos sin que haya un correspondiente rendimiento terapéutico. Dos ejemplos (17): uno, el gasto farmacéutico en Cataluña (27) es el $25 \%$ del gasto sanitario total, siendo el gasto en tratamientos para el Sistema Nervioso Central el mayor de todas las especialidades (aunque últimamente se ha contenido el gasto de forma notable, en gran parte recortando márgenes farmacéuticos); dos, el dispendio en camas psiquiátricas sigue siendo ingente, consumiendo un elevado tanto por ciento del presupuesto en Salud Mental (ya hemos señalado que es un 55-60\%, destacando las de crónicos en un $35 \%$ ).

- El modelo de atención en salud mental comunitario al que apunta la comisión para la reforma basada en la atención primaria de salud, así como en la atención especializada y los recursos socio-sanitarios en la comunidad (centros de salud mental, centros de dia, hospitales de dia...), está muy dificultado, por la saturación de estos servicios y la infradotación de esos recursos (entre todos ellos sólo alcanzan el $40 \%$ del gasto sanitario total en salud mental). 
- Los recursos comunitarios de reinserción, en el marco de todo el territorio de Cataluna, son muy deficitarios. No resulta por ello nada fácil la rehabilitación de los enfermos más graves. La escasez de esos recursos no permite una fácil externación de los pacientes ingresados ni resolver las indicaciones que se puedan realizar desde los recursos primarios especializados (los programas para TMS están por ello limitados) ni aprovechar los resultados de los programas de rehabilitación que se realizan en los recursos intermedios.

- Muchos servicios siguen girando alrededor del hospital psiquiátrico que mantiene un importante población de enfermos, a pesar de que algunos hospitales psiquiátricos han puesto en marcha proyectos de reconversión (externación de pacientes, programas de rehabilitación destinados a la reinserción en la comunidad...). Pero esos nuevos servicios se crean a veces en el interior de los hospitales psiquiátricos o están en la órbita del hospital. Mencionemos que hoy no existe ninguna propuesta así expresada de auténtica superación del hospital psiquiátrico, tal como aconsejaba la comisión para la reforma.

- Hay multiplicidad de redes paralelas (a veces diferentes proveedores se ocupan de un mismo sector); no hay un verdadero funcionamiento en red (proveedores con modelos muy variados, tendencia al funcionamiento autónomo de los hospitales generales, dificultades para la continuidad de cuidados...); las unidades de atención a toxicomanías (CAS) y las de Estimulación Precoz no están integradas en la red general; Sanidad apenas se coordina con otras áreas (Trabajo, Justicia, Educación), salvo programas puntuales; no existen propiamente Áreas de salud, etc.

- No se ha reducido la hospitalización psiquiátrica a lo imprescindible, pues el modelo hospitalario y custodial mantiene un gran peso. Se han disminuido las camas psiquiátricas, pero el reingreso de pacientes es demasiado frecuente, posiblemente debido a la escasez de recursos de reinserción y de rehabilitación activa, entre otras causas.

- Las acciones terapéuticas no se facilitan en el entorno social de los enfermos, sino muy a menudo en lugares muy alejados (entre el $60-80 \%$ de los recursos, sobre todo los especializados, están en Barcelona provincia). $Y$ no siempre los pacientes crónicos pueden beneficiarse de recursos en su entorno socio-familiar. Al contrario, gran parte del peso de los cuidados de una población con alto grado de cronicicidad recae en los familiares que en demasiadas ocasiones no disponen de recursos rehabilitadores cercanos ni de atención domiciliaria adecuada ni de redes de voluntariado a quienes recurrir.

- No se realizan suficientes programas de rehabilitación activos y menos de prevención. Hay que señalar que las primeras crisis de casos graves, que reciben atención farmacológica e ingreso psiquiátrico cuando lo precisan, 
no siempre cuentan con los adecuados programas de rehabilitación y de recursos comunitarios que evitarían su posible cronificación. Por otro lado, los programas de prevención de los trastornos mentales están poco desarrollados.

- La formación de nuevos profesionales está muy marcada por un modelo biomédico que se realiza básicamente en unidades hospitalarias y tiende a seguir líneas de investigación farmacológica. No existe un programa de formación único ni homogéneo en el sector (el Programa de Salud Mental no reglamenta la formación, aunque ayuda de alguna manera a su financiación).

- Se mantiene aún la exclusión social del enfermo mental, pues no se ha educado a la población en la comprensión de estos trastornos, sino en la clásica concepción de un trastorno crónico de causación genética a tratar farmacológicamente.

\section{Evaluación de los efectos del modelo}

Constatamos, pues, que la salud mental catalana ha experimentado cierta reforma y mejora, y que está muy alejada del antiguo modelo neuropsiquiátrico y manicomial, pero no podemos aún hablar en puridad de una decidida transformación propia de una salud mental moderna como la que propone el documento para la reforma.

Es cierto que se han desplegado mayores recursos para la red e incluso ha aumentado ligeramente el presupuesto en salud mental, pero en general se tiende a crear recursos de ámbito hospitalario o sanitario y menos a mejorar la atención especializada o a desarrollar recursos intermedios rehabilitadores y comunitarios de inserción.

Hay que resaltar además la existencia de listas de espera de entre 2-4-6 meses (según épocas) en muchos de los recursos: Centros de salud mental, Hospitales de Día, Subagudos, Larga Estancia, Unidades de psicoterapia, etc. Con la presión añadida de que el Programa de Salud Mental pone un gran énfasis en que se disminuyan los tiempos de espera, especialmente en primeras visitas.

¿Qué efectos genera este modelo? Conviene detenerse en este punto, pues es clave y decisivo, ya que los datos no explicitan claramente las consecuencias y riesgos de la aplicación del modelo actual. Pero sus consecuencias se muestran de tres maneras por lo menos: económicas, clínicas especialmente y éticas.

Para entenderlas, hemos de imaginar este modelo funcionando en vivo y en su conjunto, entendiendo que todos los factores analizados hasta ahora actúan a la vez, sumando y potenciando sus efectos. 
Entre sus consecuencias económicas sefralemos que la aplicación de este modelo mixto, pese a que intenta limitar y ahorrar los gastos en múltiples esferas (especialmente en recursos primarios, intermedios y comunitarios), no ha conseguido reducir el consumo de recursos hospitalarios y farmacológicos (los antidepresivos y neurolépticos de nueva generación han disparado el gasto (17); así, los neurolépticos están en el segundo lugar de los fármacos que han aumentado con un $29 \%$ y los antidepresivos en el cuarto con un $18 \%$, sumando entre ambos el $10 \%$ de todo el gasto farmacológico). Por un lado, porque no ha puesto decidida voluntad en ello; por otro, porque las consecuencias clínicas del modelo no lo permiten. Eso ha conllevado el efecto contrario: seguir generando una deuda importante. No se adivinan, pues, los beneficios de la privatización del sistema (más del $60 \%$ de recursos son ya privados). Además, la concertación de recursos muy heterogéneos provenientes de proveedores con modelos de atención muy diferentes ha fragmentado el sector 0 , mejor dicho, ha hecho imposible la unificación y homogeneización propia de una red de salud.

Abundemos en los riesgos del modelo mixto economicista: tendencia a favorecer a las grandes empresas del sector; estimular la libre competencia (a menudo en un mismo sector); crear un fuerte peso burocrático; tener que imponer criterios restrictivos en las estancias de recursos intermedios y otros; favorecer que los recursos de atención se transformen en empresas de gestión; dejar al albur de los proveedores la calidad de los servicios (previsión de dotaciones en función de necesidades, análisis de carencias y consiguiente evaluación), etc.

Para captar los riesgos y consecuencias clínicas (gráfico 4) diferenciaremos dos niveles:

-Pacientes afectos de trastorno mental menos grave: la reducción del tiempo de las visitas, la baja frecuencia de éstas, el incremento de psiquiatras de la red a costa de otros profesionales, el fácil recurso a la receta sin limitaciones, los pocos recursos psicoterápicos, el poco tiempo de que disponen los médicos de cabecera (a veces poco más pueden hacer que dar fármacos y solicitar pruebas)... tienden a medicalizar y psiquiatrizar el trastorno menos grave comportando el riesgo de cronificarlo.

Mención aparte merece el ámbito infanto-juvenil, donde la detección a tiempo puede facilitar la prevención y evitar trastornos o cronicidades en la edad adulta. En este campo, donde se ha avanzado mucho, siguen siendo una importante deficiencia los recursos destinados a la atención en crisis.

-Pacientes afectos de trastorno mental grave o severo: por un lado, la escasez de recursos de reinserción y rehabilitación, la insuficiente aún dotación de los programas TMS y PSI, los pobres recursos comunitarios y redes de voluntariado y, por otro, la tendencia a la contención hospitalaria y al recurso farmacológico, la insuficiente 
INFORMES

coordinación de la red ( $\mathrm{y}$ de la continuidad de cuidados), la depositación en las familias... tienden a facilitar la cronificación y el deterioro de muc

-Las consecuencias éticas se vislumbran en la introducción de criterios de valor mercantilista en un ámbito donde debería primar la atención al enfermo, su capacitación y su consideración como ciudadano de pleno derecho de la sociedad.

\section{Análisis final}

¿Cuál es, pues, el estado de la reforma en Cataluña? A nuestro criterio, si lo valoramos en el momento presente y en relación con las líneas de la reforma, nos encontramos frente a una reforma heterogénea caracterizada por logros importantes $y$ por marcadas debilidades, con fuertes desequilibrios tanto de recursos como territoriales, lastrada por el modelo mixto economicista de gestión, que tiene su mayor peso en los recursos sanitarios psiquiátrico-hospitalarios y su mayor fragilidad en la infradotación de recursos comunitarios (atención especializada y apoyo a primaria, rehabilitadores, psicoterápicos, de reinserción...) y en la inadecuada planificación de los recursos, lo que genera riesgos y consecuencias económicas, clínicas y éticas.

¿Qué posibilidades de reforma tiene entonces ese modelo catalán? ¿Hacia dónde puede dirigirse? Podemos considerar que hay muchos puntos y líneas para la mejora del modelo de atención en salud mental catalán, pese a las dificultades señaladas. Y sabemos que sigue vigente la semilla que no hace tantos años hizo de Cataluña una de las pioneras de la reforma. De tanto en tanto en nuestra tierra vuelve a resurgir ese espíritu $(10,12,23$, 28, 29, 30, 31, 32, 33, 34). Así pues, no podemos ser pesimistas, pero hemos de cuidar mucho los caminos futuros. Hay ciertamente amenazas, pero también oportunidades muy a tener en cuenta, y desde todos los ámbitos (gráfico 5). Precisamos, eso sí, una voluntad colectiva de llevarlos a cabo, entre todos, asociaciones, políticos, profesionales, familiares y los propios enfermos.

Necesitaremos seguramente tiempo y cuidar al máximo dichas oportunidades, para ganar la lucha mundial que se ha entablado para tener una atención de calidad en salud mental y dejar ya de lado la exclusión que tanto marcó a los enfermos. Tiempo y capacidad de espera, pues como dice el poeta Mario Benedetti hablando de otras batallas: «...la victoria crecerá despacio, como siempre han crecido las victorias».

Ese es el reto. Hacia ello apuntamos.

Nuestro agradecimiento a todos los profesionales que han colaborado en este trabajo. Sin ellos, no hubiera sido posible. 


\section{TABLA 1. CRONOLOGÍA HISTÓRICA}

\begin{tabular}{|c|c|}
\hline 1914 & Creación mancomunidad cuatro diputaciones \\
\hline 1931 & Decreto asistencia psiquiátrica \\
\hline 1936 & $\begin{array}{l}\text { Se crea Conselleria Sanidad y Asistencia Psiquiátrica Proyecto piloto } \\
\text { red asistencial comunitaria }\end{array}$ \\
\hline 1967 & Proyectos comunitarios desde la Diputación \\
\hline 1970 & Decreto de comisión técnica asistencia psiquiátrica \\
\hline 1975 & $\begin{array}{l}\text { Plan Asistencia Psiquiátrica (Sector, H.Día...) Van surgiendo centros } \\
\text { de Higiene Mental }\end{array}$ \\
\hline 1978 & Creación Insalud: no asume psiquiatria \\
\hline 1981 & Nuevo plan Asistencia Psiquiátrica \\
\hline $1979-1981$ & Asunción de transferencias de Seguridad Social \\
\hline $1981-1983$ & Despliegue mapa sanitario \\
\hline 1981 & $\begin{array}{l}\text { Se crea el Institut Català de Salud Red Hospitalaria de Utilización } \\
\text { Pública (XHUP) }\end{array}$ \\
\hline 1985 & Comisión para la reforma - Jornadas de la Diputación \\
\hline 1986 & Ley General Sanidad \\
\hline 1990 & Inicia traspaso competencias de Diputación a Generalitat \\
\hline 1991 & $\begin{array}{l}\text { LLOSC: Ley General de Ordenación Sanitaria Se crean Servei Català } \\
\text { de Salut y Pla de Salut Mental }\end{array}$ \\
\hline $1987-89-91$ & $\begin{array}{l}\text { Red de toxicomanias, red de salud mental infanto-juvenil, } \\
\text { red de centros de día }\end{array}$ \\
\hline 1999 & Decreto de creación Red de Salud Mental \\
\hline
\end{tabular}


INFORMES

\section{GRÁFICO 1}

MODELO TRADICIONAL (centrado en el Hospital Psiquiátrico y consulta tradicional)

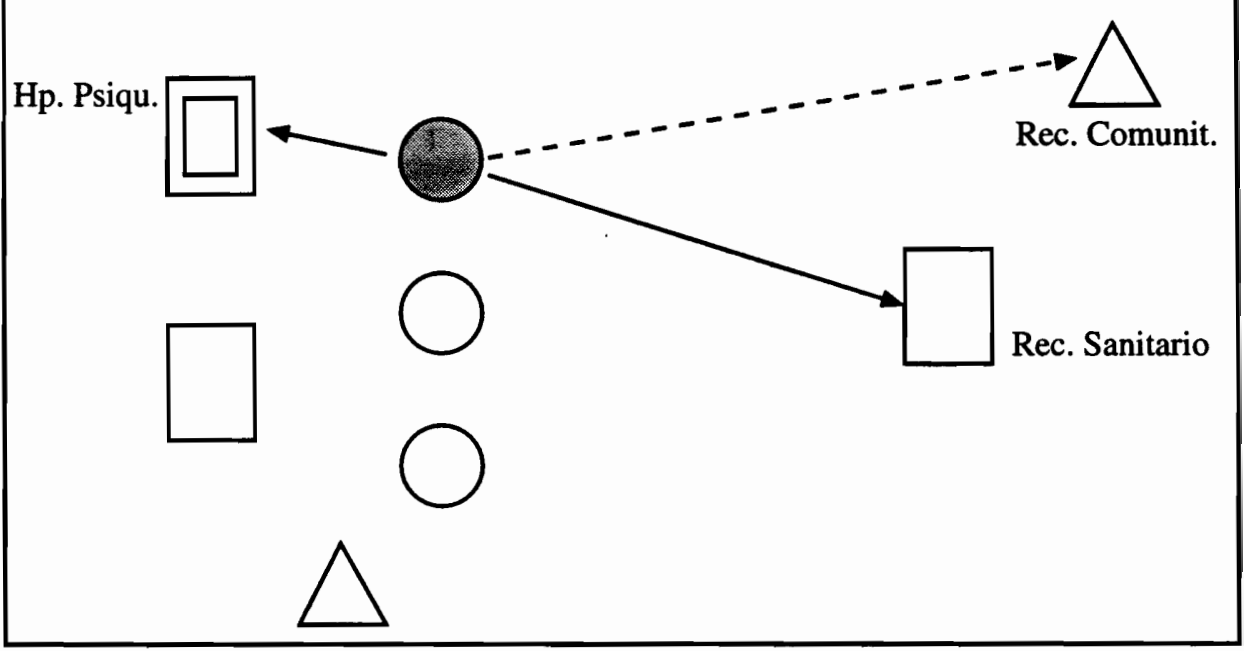

MODELO REFORMA (basado en redes sanitarias y comunitarias interconectadas)

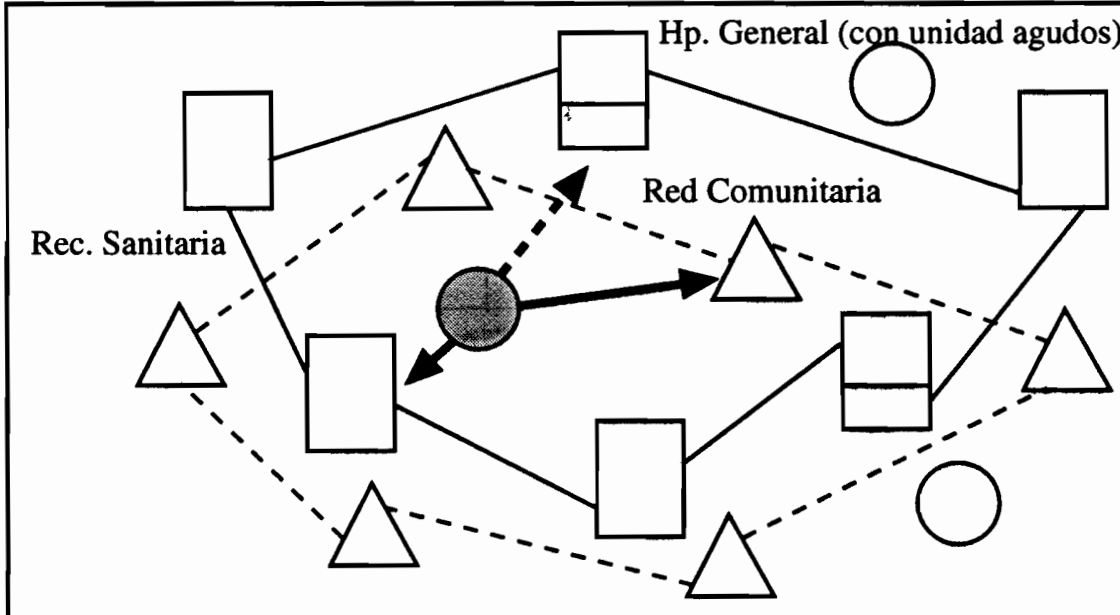

Recurso sanitario

Hosp. Psiquiátrico
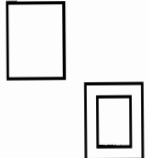

Nucleo familiar

Rec. comunitario

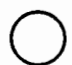

$\triangle$

Unidad Agudos Hosp. General

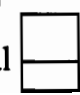


TABLA 2. MAPA DE RECURSOS(2000)

\begin{tabular}{|c|c|c|c|c|c|c|}
\hline & BARCELONA & GERONA & LÉRIDA & TARRAGONA & TOTAL & PLA ZAS \\
\hline CSMA & $46+5 c$. Perif & $6+1 c$. Perif & $6+$ Ic.Perif & 8 & $66+7 \mathrm{cp}$ & \\
\hline CSMIJ & $29+12$ c.p & $2+4$ c.p & $1+6$ c. $p$ & $1+6$ c.p. & $32+28 \mathrm{cp}$ & \\
\hline H.DÍA ADUL* & $4+3$ h.h.g ** & No & 1 & No & 8 & 276 \\
\hline H. DIA INF * & $2+2$ h.h.g & 1 h.h.g & 1 h.h.g & No & 6 & \\
\hline H.DIA ADOL & 1 & No & No & 1 & 2 & 124 \\
\hline COM. TERAP & 3 & No & No & No & 3 & \\
\hline C DIA ADUL & 32 & 6 & 1 & 3 & 42 & 1177 \\
\hline C D INF & 7 & No & No & No & 7 & 232 \\
\hline UNI POLIVAL & 1 & No & No & No & 1 & \\
\hline UCA & 1 & No & No & No & 1 & 48 \\
\hline URPI & $2+1$ c.h.g $* * *$ & 1 c.h.g & lc.h.g & No & 5 & \\
\hline CAS & & & & & & 54 \\
\hline \multicolumn{7}{|c|}{ UNIDADES DE HOSPITALIZACIÓN } \\
\hline Agudos & 15 & 1 & 1 & 1 & 18 & 788 \\
\hline Subagudos & 9 & 1 & 1 & 1 & 12 & 394 \\
\hline Crón (MILLE) & 8 & 1 & 5 & 2 & 16 & 3325 \\
\hline \multicolumn{6}{|c|}{ Agudos en Hosp. General } & 287 \\
\hline \multicolumn{6}{|c|}{$\%$ águdos en Hosp. Psiquiátrico } & $68 \%$ \\
\hline
\end{tabular}

* No se han incluido las Unidades Especializadas (por ej. las de atención a anorexia que son 3)

**Hospital de Día consistente en una Unidad en Hospital General

***Unas camas en Hospital General

\begin{tabular}{|l|r|}
\hline PISOS PROTEGIDOS (plazas) & $160-170$ \\
\hline RESIDENCIAS (plazas) & $100-130$ \\
\hline TALLER PROTEGIDO (plazas) & 150 \\
\hline CLUB SOCIO-TERAPÉUTICO & 3 \\
\hline
\end{tabular}


INFORMES

\section{GRÁFICO 2}

\section{PRESUPUESTO TOTAL SALUD MENTAL}

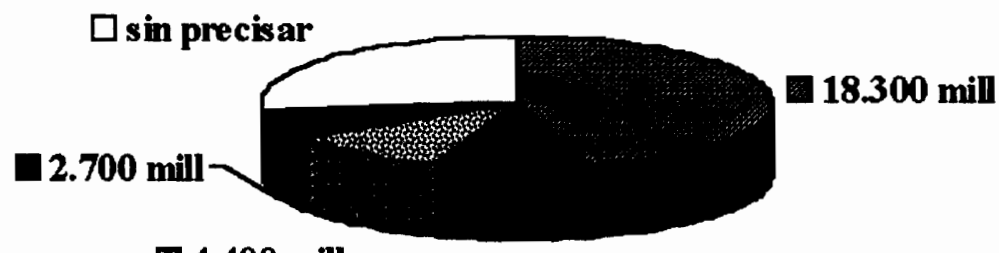

4.400 mill 4000 mill

O Programa Salud Mental Dsiquiatría Hospital Gen eral a Psicogeriatria

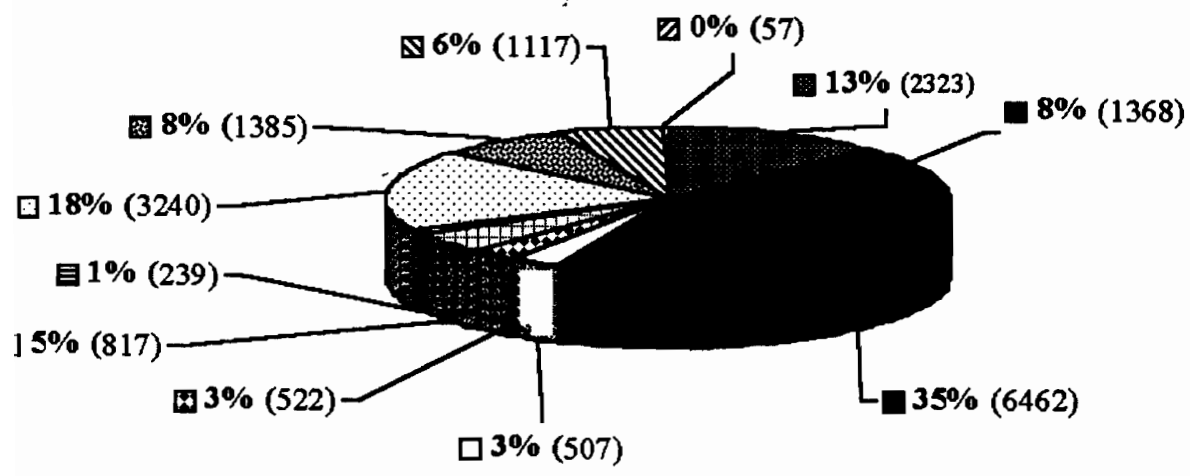

Hosp italización águdos

口 Hosp ital dia adultos

B Formación

Centro de día adulos
Subragudos

Hospital dia infentil

DCSMA

Centro de día infantil
Larga estancia

由 Programas especiales

GSMLJ 
GRÁFICO 3. Fortalezas y debilidades

Desequilibrio hacia fortalezas $(-)$

\section{Debilidades}

Redes descoordinadas, paralelas

Mínima infraestructura recursos comunitarios (pisos prot., residencias, talleres, voluntariado)

Bajo presupuesto en salud mental

Infradotación recursos intermedios

Formación en S. M biomédica y hospitalaria

Predominio recursos Barcelona provincia

Precariedad laboral profesionales

Poca sensibilización sociedad

\section{Fortalezas (一)}

Influencia grandes proveedores Gran presencia Hosp. Psiquiátrico FunC: autónomo Hosp. General Tendencia favorecer recursos Hosp. Gran consumo farmacéutico Importante peso burocrático Modelo mixto liberal

Fortalezas (+) equilibran

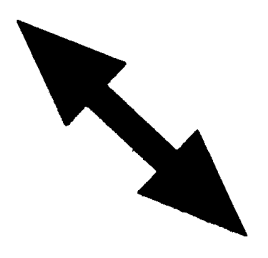

Fortalezas (+)

Profesionales comprometidos Centros S. M en comunidad Buena atención primaria gral. Asociaciones usuarios Asociaciones profesionales Sectores con program. calidad Planes reconvers. H. Psiquiátr. Sectores social. sensibilizados 
INFORMES

\section{GRÁFICO 4. Riesgos y consecuencias clínicas}

Fragmentación y descoordinacion

Farmacología incisiva

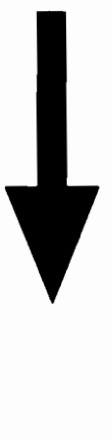

Contención hospitalaria

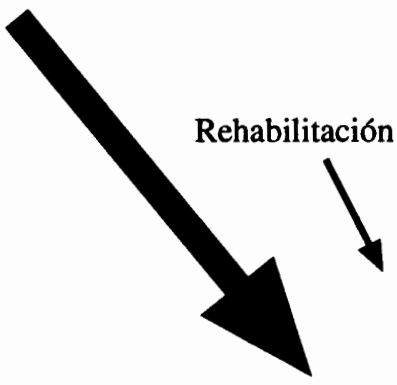

Rec. comunitarios

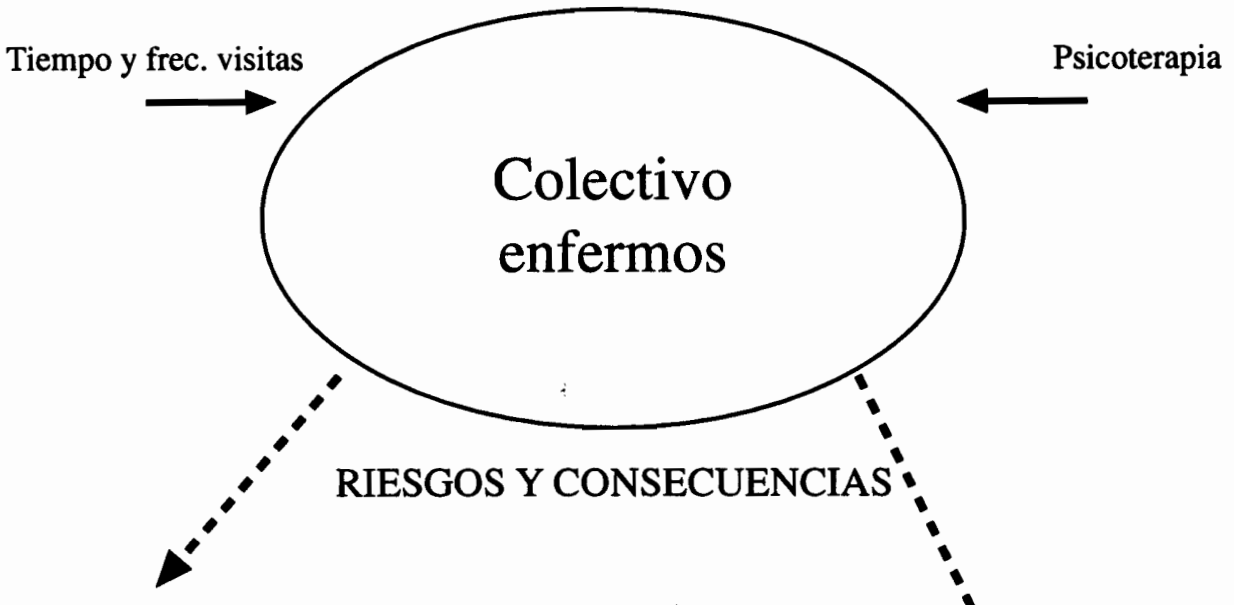

MEDICALIZACIÓN Y PSIQUIATRIZACIÓN TRASTORNO MĘNOS GRAVE

Debilidad recurso 


\section{GRÁFICO 5. Amenazas y oportunidades}

\section{Amenazas}

Presupuesto bajo, poca contención gasto

Saturación servicios

Modelo mixto de gestión, liberal

Insuficiente coordinación circuito

Persistencia modelo hospitalario psiquiátrico

Cambio en formas, igual fondo

Gran peso monopolio farmacéutico

Privatización red

\section{Oportunidades}

Sensibilidad en S. M de administración

Peso de asociaciones concienciadas

Voluntad política

Voluntad cambio en profesionales

Persistencia espíritu de reforma

Buen nivel técnico profesional

Consenso en el modelo comunitario 
INFORMES

\section{BIBLIOGRAFÍA}

1. Diéguez-Liria, «La reforma psiquiátrica en España: aspectos diferenciales», Revista de administración sanitaria, vol II, n. ${ }^{\circ}$ 5, 1998

2. J. García, A. Espino, L. Lara, La psiquiatria en la España de fin de siglo, Madrid, Ed. Diaz de Santos, 1998

3. Varios autores, "Informe sobre la Salud Mental en España» (entrevista de la Junta directiva de AEN con la Ministra de Sanidad), Madrid, 2001

4. González de Chaves, La transformación de la asistencia psiquiátrica, Madrid, Ed. Mayoría, 1980

5. V. Aparicio, «El declinar de una reforma devaluada», revista AEN, Octubre/ Diciembre 1999, número 72

6. Amalia Tesoro, «El porvenir de la reforma psiquiátrica en Andalucía; retos y perspectivas futuro" (ponencia del VII congreso de la AAN), Sevilla, 2001

7. Varios autores, La reforma psiquiátrica en Andalucia, 1984-1990, (Libro monográfico), Edita IASAM, 1998

8. Varios autores, Memoria SESPA (Servicios salud Principado Asturias), Libro monográfico, 1998-1999

9. J. Lázaro, «Historia de la Asociación Española de Neuropsiquiatría», Junio-Septiembre 2000, número monográfico de la revista $\mathrm{AEN}, \mathrm{n} .^{\circ} 75$

10. Varios autores, «Primeres jornades sobre l'ordenació de l'assistència psiquiàtrica a Catalunya», organització: Diputacions de Barcelona, Lleida, Girona i Tarragona, Dep. Sanitat, Barcelona, 1985

11. Varios autores, «Documento para la reforma psiquiátrica» (Informe de la comisión ministerial), Dep. Sanidad, Madrid, 1985

12. Servei Català de la Salut, Document de bases per a la reordenació de la Salut Mental, (dos volúmenes), Barcelona, 1997

13. Servei Català de la Salut, «Pla de Salut de Catalunya» (1999-2001), Departament de Sanitat $\mathrm{i}$ Seguritat Social, Generalitat de Catalunya, Barcelona

14. Iván de la Mata Ruiz, "El mito de la gestión privada: el modelo catalán de salud mental», Psiquiatria Pública, vol 11, n. ${ }^{\circ}$ 3, Madrid 1999

15. A. Espino, «Fundaciones sanitarias y privatización», Diario El País (16 de Mayo del 2000), Barcelona

16. Varios autores, Programa de Atención Especifica al Trastorno Mental Severo, Servei Català de la Salut, 1997

17. Servei Català de la Salut, Memoria d'activitat «La xarxa sanitaria d'utilització pública, Servei Català de la Salut, 1999

18. Servei Català de la Salut, Reordenació dels serveis d'atenció psiquiàtrica i salut mental a la ciutat de Barcelona. Servei Català de la Salut, Noviembre 2000.

19. Institut Català d'Assistència i Serveis Socials, «Pla d'atenció i integració social de les persones amb malaltia mental», Document de Benestar Social, 1999-2001

20. Servei Català de la Salut, Decreto ley sobre «La Xarxa de Centres de Salut Mental d'utilització pública de Catalunya», Diari Oficial de la Generalitat de Catalunya, Barcelona 1999

21. Varios Autores, Hacia una atención comunitaria de calidad, Cuadernos técnicos de la AEN n. ${ }^{\circ}$, Madrid 2000 
22. Servei Català de la Salut, "Sectorizació» (documento), Barcelona, Servei Català de la Salut, 2000

23. Varios autores, «Encuesta sobre el papel del psicólogo clínico en equipos salud mental», Documento de la Comisión salud mental del Colegio de Psicólogos, Barcelona, 2001

24. Servei Català de la Salut (Gerencia d'atenció psiquiàtrica). «Distribució de la població de Catalunya per centres de salut mental». Barcelona 1998.

25. Ministerio del Interior, Memoria 1999: Plan Nacional de Drogas. Madrid, 2000

26. Varios autores, «Rehabilitación comunitaria en Salud Mental» (documento), Servei Català de la Salut, Barcelona, Julio 1999

27. Diario El País, «La fáctura farmacéutica», 4 de septiembre de 2000

28. Varios autores, Primeres Jornades de l'Associació Catalana de Professionals d'Assistència Pública en Salut Mental Edita ACPAPSM, Barcelona, 1993

29. Asociación familiares enfermos mentales cataluña, «Informe sobre la problemática de la Salud Mental en Cataluña», Diari de sessions parlament de Catalunya, Barcelona, 1998

30. Varios autores (Acsam, Forum, Unió Catalana de Hospitales), «Dossier Día de la Salud Mental: manifiesto e informe» (documento), Barcelona, 1996

31. Varios autores, «Manifest de coordinadora CSMA en ocasió del dia mundial de la salut» (documento), Barcelona 2001

32. Varios autores, «Manifest plataforma d'entitats per el dia mundial de la salut mental» (documento), Barcelona 2001

33. José Garcia Ibañez, «La situación en Cataluña» (documento), Sete, 1999

34. Varios autores, «La evolución del modelo asistencial en Cataluña a debate», documento de la comisión gestora de la Asociación Catalana de Profesionales de Salud Mental, Barcelona, 1999

Fecha de recepción: 9/4/01

Correspondencia a:

Asociación Catalana de Profesionales de Salud Mental

C/. Providencia, 42 - 08024 Barcelona 